\title{
Rapid and Reproducible MALDI-TOF-Based Method for the Detection of Vancomycin-Resistant Enterococcus faecium Using Classifying Algorithms
}

\author{
Ana Candela ${ }^{1,2, *}++^{(D}$, Manuel J. Arroyo ${ }^{3,+}\left(\mathbb{D}\right.$, Ángela Sánchez-Molleda ${ }^{3} \mathbb{D}$, Gema Méndez ${ }^{3}$, Lidia Quiroga ${ }^{1,2}$, \\ Adrián Ruiz ${ }^{1,2}$, Emilia Cercenado ${ }^{1,2,4,5}$ (D), Mercedes Marín ${ }^{1,2,4}$, Patricia Muñoz ${ }^{1,2,4,5}$ (D), Luis Mancera ${ }^{3}$, \\ David Rodríguez-Temporal ${ }^{1,2, * \mathbb{D}}$ and Belén Rodríguez-Sánchez ${ }^{1,2} \mathbb{D}$
}

check for updates

Citation: Candela, A.; Arroyo, M.J.; Sánchez-Molleda, Á.; Méndez, G.; Quiroga, L.; Ruiz, A.; Cercenado, E.; Marín, M.; Muñoz, P.; Mancera, L.; et al. Rapid and Reproducible MALDI-TOF-Based Method for the Detection of Vancomycin-Resistant Enterococcus faecium Using Classifying Algorithms. Diagnostics 2022, 12, 328. https://doi.org/ 10.3390/diagnostics12020328 Academic Editors: Andreas Kjaer and Raul Colodner

Received: 29 December 2021 Accepted: 24 January 2022 Published: 27 January 2022 Publisher's Note: MDPI stays neutral with regard to jurisdictional claims in published maps and institutional affiliations.

Copyright: (C) 2022 by the authors. Licensee MDPI, Basel, Switzerland. This article is an open access article distributed under the terms and conditions of the Creative Commons Attribution (CC BY) license (https:// creativecommons.org/licenses/by/ $4.0 /)$.
1 Clinical Microbiology and Infectious Diseases Department, Hospital General Universitario Gregorio Marañón, 28007 Madrid, Spain; lidia.quirogam@gmail.com (L.Q.); arauko.bmcelta@gmail.com (A.R.); emilia.cercenado@salud.madrid.org (E.C.); mercedes.marinar@salud.madrid.org (M.M.); patricia.munoz.garcia@salud.madrid.org (P.M.); mbelen.rodriguez@iisgm.com (B.R.-S.)

2 Instituto de Investigación Sanitaria Gregorio Marañón, 28007 Madrid, Spain

3 Clover Bioanalytical Software, Av. del Conocimiento, 41, 18016 Granada, Spain; manuel.arroyo@cloverbiosoft.com (M.J.A.); angelamolleda97@gmail.com (Á.S.-M.); gema.mendez@cloverbiosoft.com (G.M.); luis.mancera@cloverbiosoft.com (L.M.)

4 CIBER de Enfermedades Respiratorias (CIBERES CB06/06/0058), 28029 Madrid, Spain

5 Medicine Department, School of Medicine, Universidad Complutense de Madrid, 28040 Madrid, Spain

* Correspondence: acandelagon@gmail.com (A.C.); david.rodriguez@iisgm.com (D.R.-T.); Tel.: +34-91-426-9595 (D.R.-T.)

+ These authors contributed equally to this work.

\begin{abstract}
Vancomycin-resistant Enterococcus faecium represents a health threat due to its ability to spread and cause outbreaks. MALDI-TOF MS has demonstrated its usefulness for E. faecium identification, but its implementation for antimicrobial resistance detection is still under evaluation. This study assesses the repeatability of MALDI-TOF MS for peak analysis and its performance in the discrimination of vancomycin-susceptible (VSE) from vancomycin-resistant isolates (VRE). The study was carried out on protein spectra from 178 E. faecium unique clinical isolates-92 VSE, 31 VanA VRE, 55 VanB VRE-, processed with Clover MS Data Analysis software. Technical and biological repeatability were assayed. Unsupervised (principal component analysis, (PCA)) and supervised algorithms (support vector machine (SVM), random forest (RF) and partial least squares-discriminant analysis (PLS-DA)) were applied. The repeatability assay was performed with 18 peaks common to VSE and VRE with intensities above $1.0 \%$ of the maximum peak intensity. It showed lower variability for normalized data and for the peaks within the 3000-9000 m/ $z$ range. It was found that $80.9 \%$, $79.2 \%$ and $77.5 \%$ VSE vs. VRE discrimination was achieved by applying SVM, RF and PLS-DA, respectively. Correct internal differentiation of VanA from VanB VRE isolates was obtained by SVM in $86.6 \%$ cases. The implementation of MALDI-TOF MS and peak analysis could represent a rapid and effective tool for VRE screening. However, further improvements are needed to increase the accuracy of this approach.
\end{abstract}

Keywords: enterococci; vancomycin resistance; MALDI-TOF; mass spectrometry; peak analysis

\section{Introduction}

Enterococcus faecium are Gram-positive, non-spore forming, facultative anaerobic cocci that can be found as part of the microbiota of the human gastrointestinal tract [1]. Because of its genomic plasticity and its adaptation to harsh conditions, E. faecium has become a health threat due to its ability to rapidly spread and cause outbreaks in hospital settings [2,3]. Adding up to the intrinsic antibiotic resistance displayed by E. faecium, infections caused by strains with acquired resistance to certain antimicrobials are of special interest [4]. In 
the past years, vancomycin resistance in E. faecium has been a major concern owing to its rise and rapid spread of high-risk clones among hospitalized patients [5]. Therefore, E. faecium was included in the ESKAPE group of highly resistant microorganisms because of its ability to "escape" the action of conventional treatments [6]. Vancomycin resistance is by far the biggest threat regarding enterococci, mainly E. faecium, due to its position as first-line treatment for multidrug-resistant enterococcal infections $[7,8]$.

Susceptibility to vancomycin can be routinely determined in the clinical microbiology laboratory using two approaches: (i) phenotypically, by the standard broth antimicrobial susceptibility testing microdilution method or by gradient diffusion, or (ii) genotypically, by amplification of the van $A / \operatorname{van} B$ genes and subsequent analysis of the specific amplicons $[9,10]$. The first approach has a turnaround time of approximately 2 days. Although the implementation of molecular methods provides final results in $1-3 \mathrm{~h}$ after isolation in culture, its cost in laboratory reagents is high.

While the usefulness of matrix-assisted laser desorption/ionization time-of-flight mass spectrometry (MALDI-TOF MS) in the microbiology laboratory for bacterial identification is settled, its implementation for antimicrobial resistance detection is not well standardized yet [11].

In the last years, MALDI-TOF MS has been proposed as a fast and cost-efficient method for the detection of some antimicrobial resistance mechanisms, such as $\beta$-lactamase activity [12], discrimination of methicillin-resistant $S$. aureus [13] or the detection of the cfiA gene in B. fragilis [14,15]. As for vancomycin resistance in Enterococcus, a few studies have been published recently but with variable results [16-18].

The use of MALDI-TOF MS in the clinical microbiology laboratory will be of interest as a rapid approach for the differentiation of VRE from VSE, based on their mass spectra protein profile.

The main objective of this study was the development of a MALDI-TOF-based classifying algorithm for the discrimination of vancomycin-resistant E. faecium (VRE) from vancomycin-susceptible E. faecium (VSE).

\section{Materials and Methods}

\subsection{Bacterial Strains}

A total of $n=178$ E. faecium strains were included in the study and considered as the "classification set" (92 VSE, 31 VanA VRE, 55 VanB VRE). The isolates were collected consecutively throughout the years 2017 to 2019 from clinical samples of patients admitted at Hospital General Universitario Gregorio Marañón in Madrid (HGUGM) (Figure 1). Only one sample per patient was selected.

Strains were selected from blood cultures $(n=95)$ and rectal swabs $(n=83)$ (Table S1). Clonality was clinically and epidemiologically discarded by analyzing the date, patient location and department where the inpatients were located. No clinical outbreak was detected during the period of study. All strains were isolated from inpatient clinical samples, characterized and kept frozen at $-80{ }^{\circ} \mathrm{C}$ for further analysis. For this study, samples were thawed and cultured overnight at $37^{\circ} \mathrm{C}$ in Columbia blood agar.

\subsection{Antibiotic Susceptibility Testing for Vancomycin}

Antimicrobial susceptibility testing was performed with the automated microdilution method Microscan ${ }^{\circledR}$ System (Beckman-Coulter, Brea, CA, USA) using PM33 panels following the manufacturer's guidelines. Vancomycin and teicoplanin breakpoints were stablished as indicated by the EUCAST (2021) v. 11. The results obtained were confirmed by real-time PCR for the amplification of the vanA and vanB genes [9]. In addition, the presence of the vancomycin resistance genes was confirmed a second time by the implementation of the commercial Xpert ${ }^{\circledR}$ vanA/vanB cartridges (Cepheid, Sunnyvale, CA, USA). 


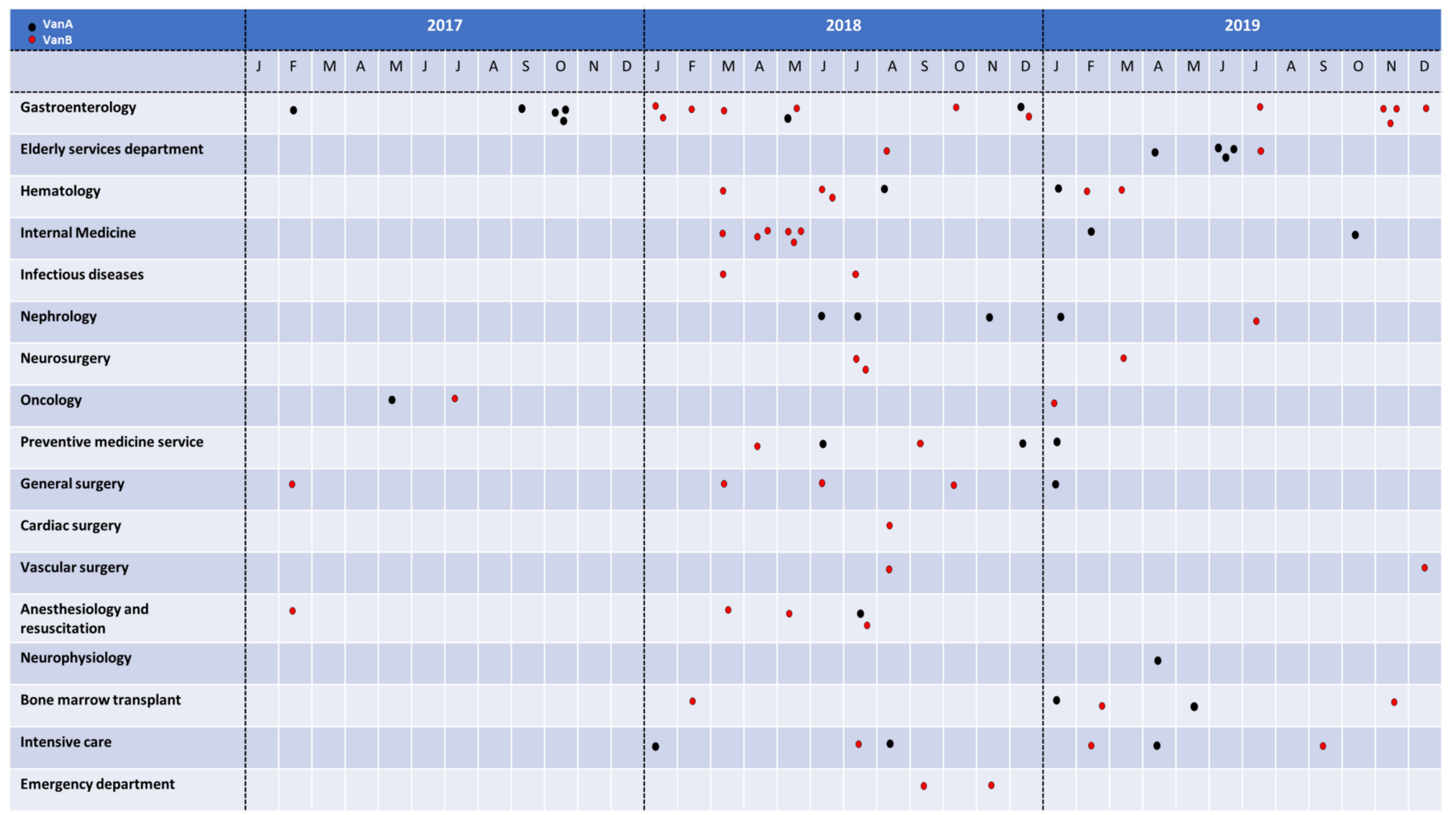

Figure 1. Distribution of the E. faecium isolates analyzed in this study by hospital departments and date. VanA E. faecium isolates are shown with black dots and VanB isolates with red dots.

\subsection{Identification of the Isolates by MALDI-TOF MS}

Bacterial strains were analyzed by MALDI-TOF MS in an MBT Smart MALDI Biotyper (Bruker Daltonics, Bremen, Germany) using the updated database containing 9957 mass spectra profiles (MSPs). A few bacterial colonies of each enterococcal isolate were spotted onto the MALDI target plate. On-plate protein extraction was performed by applying $1 \mu \mathrm{L}$ of formic acid on each target spot and letting it dry at room temperature before adding $1 \mu \mathrm{L}$ of HCCA matrix solution (Bruker Daltonics, Bremen, Germany), following the manufacturer's instructions. Spectra were acquired in positive mode in the range of 2000 to $20,000 \mathrm{Da}$, applying default settings [19].

\subsection{Spectra Acquisition and Pre-Processing}

Each isolate was analyzed in two different spots from the MALDI target plate, and each spot was read twice, obtaining four spectra per strain [20]. Protein spectra were visually inspected with FlexAnalysis (Bruker Daltonics, Bremen, Germany) and aligned with the genus-specific peak at $4428 \mathrm{~m} / \mathrm{z}$, present in all isolates $[16,21]$. Outlier spectra and zero lines were discarded.

After the previous screening, protein spectra were processed with Clover MS Data Analysis software (https: / / platform.clovermsdataanalysis.com, accessed on 30 November 2021, Clover Biosoft, Granada, Spain). For classification purposes, peak matrices were generated in the range of 2000 to $20,000 \mathrm{~m} / \mathrm{z}$. For this goal, pre-processing was performed as follows: a Savitzky-Golay filter (window length 11; polynomial order 3) was applied for smoothing spectra, and then the baseline was removed by the top-hat filter method (factor 0.02).

Processed spectra were aligned using the following method: replicates within the same spot were aligned to create an average spectrum per spot (shift medium; linear mass tolerance $2000 \mathrm{ppm}$ ). Then, average spectra from each replicated spot were aligned, and thus one average spectrum per sample was obtained. Finally, average spectra from different isolates were aligned together. 


\subsection{Repeatability Test}

A repeatability study was performed to determine the robustness of MALDI-TOFMS-based bacterial classification. For this purpose, 20 different E. faecium isolates (VSE, $n=10$; and VRE strains, $n=10,5$ VanA VRE and 5 VanB VRE) were randomly selected and considered as the "repeatability set" (Table S2). For intra-spot repeatability, two spectra per spot were acquired, whilst for inter-spot repeatability (or technical repeatability), an average spectrum was built from each spot. Finally, each isolate was subcultured during three consecutive days, and their average spectra were compared in order to evaluate the biological (inter-day) repeatability of the method (Figure 2). This methodology has been described by Oberle et al., 2016 [20]. A final number of 12 spectra per isolate was obtained.

INTRA-SPOT

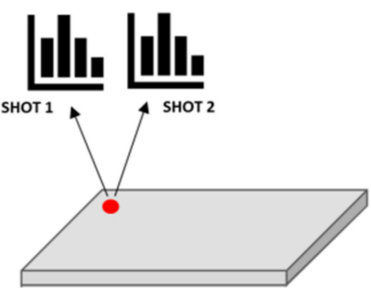

DAY 1
INTER-SPOT (Technical repeatability)

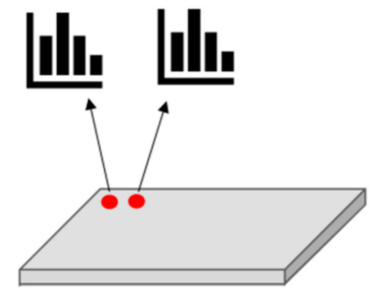

DAY 2

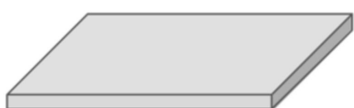

DAY 3

INTER-DAY (Biological repeatability)

INTER-DAY

Figure 2. Graphic representation of 12 replicates spectra for each isolate: 2 spectra are acquired per spot; 2 spots analyzed each day and compared for 3 consecutive days.

All spectra were pre-processed using Clover MS Data Analysis Software following the pipeline described in Section 2.4. The post-processed spectra (smoothed and baseline removed) were aligned (shift medium; linear mass tolerance $2000 \mathrm{ppm}$ ) to obtain an average spectrum for each spot. This process was repeated to obtain a single average spectrum per day for each isolate.

Once the average Day 1 spectra from all isolates were aligned, a first assay was performed to identify all common peaks and establish their characteristic peak profiles. Peak finding was carried out by applying a threshold filter (0.01), so peaks with less than $1.0 \%$ of the maximal intensity recorded were discarded. Group-specific peaks were then searched in the repeatability set by the mass position method (constant mass tolerance $0.2 \mathrm{Da}$; linear mass tolerance $500 \mathrm{ppm}$ ) at the shot, spot and day levels.

The coefficient of variation $(\% \mathrm{CV})$ of the intensities registered for each of these common peaks was calculated from raw spectra and from spectra normalized with the TIC method in two different ways: (i) normalizing by TIC the peaks previously found in raw spectra (pTIC) and (ii) normalizing first the entire spectra by TIC and then finding the common peaks (TICp). Results from both methods and from raw data were compared for intra-spot, technical and biological repeatability.

In addition, Pearson correlation coefficient $(p)$ was applied to protein spectra from VRE and VSE isolates to measure how biological variation could affect the discrimination of these two groups and how reproducibly the discrimination could be performed.

This peak study also included the calculation of arithmetic and post-alignment means and the subsequent comparison between them. The arithmetic means for spots and days were calculated directly from the intensity peak values of each shot. On the other hand, the post-alignment means were calculated automatically with Clover MS Data Analysis software after replicated average spectra were aligned. Both mean values were compared at spot and day levels. The assay was repeated without normalization and for both TIC methods. Thereby, the automatic alignment and replicate software process could 
be compared with the ideal arithmetic model in the three normalization cases. Once the Shapiro-Wilk and Levene test was applied to verify the normal distribution and the homoscedasticity of the data, a Student's t-test was performed to verify whether the null hypothesis of equality of means was fulfilled for the three normalization methods.

\subsection{Classification of E. faecium Isolates Based on Their MALDI-TOF MS Protein Spectra}

Protein spectra from E. faecium isolates were acquired as described in Section 2.4. Clover MS Data Analysis Software was applied to differentiate (1) susceptible from resistant E. faecium strains and (2) isolates hosting van $A$ and van $B$ resistance genes. For this purpose, three different methods were evaluated: (i) a "full-spectrum method", in which the matrix obtained included all peak intensities from the spectrum separated by $0.5 \mathrm{Da}$ regardless of their intensity. Their intensities were then normalized by total ion current (TIC) normalization. The other two methods used a peak matrix generated by a "threshold method", in which only peaks with intensities above $1.0 \%$ of the maximum peak intensity ( 0.01 factor) were chosen. The difference in the last two methods was the order in which TIC normalization was applied: (ii) before (TICp) or (iii) after (pTIC) searching peaks by the threshold method.

The three peak matrices described above were used as input data for three different supervised machine learning algorithms: partial least squares-discriminant analysis (PLS$\mathrm{DA}$ ), support vector machine (SVM) and random forest (RF). These algorithms were first tested for the discrimination VSE and VRE isolates and, secondly, for the differentiation of VRE isolates hosting van $A$ and van $B$ genes.

Internal validation of the results provided by each algorithm was assayed using $\mathrm{k}$-fold cross validation $(k=10)$ as previously described [22]. Briefly, data were randomly split into 10 data subsets of the same size. The algorithms were trained with nine of them and the remaining subset was used as a test set for internal validation. This process was iterated 10 times (once for each of the 10 subsets) and the accuracy rate of the classification was recorded [23].

Furthermore, a search for potential biomarkers was performed to use group-specific protein peaks as markers for the correct classification of VanA and VanB VRE and VSE strains. For this purpose, 178 average spectra (one per sample) from Day 1 were considered as input data for the Biomarker Analysis application within Clover MS Data Analysis software. The threshold method was applied as explained above, and peaks were merged with $0.5 \mathrm{Da}$ and $300 \mathrm{ppm}$ as constant and linear mass tolerance, respectively.

\section{Results}

\subsection{Identification by MALDI-TOF MS}

All isolates $(n=178)$ included in this study were correctly identified as E. faecium by MALDI-TOF MS with score $\geq 2$.0. Identifications for this microorganism were consistent along the top 10 identifications provided by MALDI-TOF MS since this is a common pathogen well represented in the updated commercial library.

\subsection{Repeatability Study}

When the different methods for peak finding described before were applied to the Day 1 spectra from the "classification set", a total of 18 common peaks were found in all E. faecium (VSE and VRE) protein spectra with intensities above $1.0 \%$ of the maximum peak intensity (Table 1). The CV means of the intensities from these 18 common peaks were compared with the peaks present in the spectra from the repeatability set at intra-spot, inter-spot and inter-day levels.

Comparing the means of the CVs in the repeatability set showed lower $\mathrm{CV}$ values at the intra-spot level than at the inter-spot and inter-day levels. For non-normalized raw data, the average $C V$ s were $C V_{\text {intra-spot }}=15.35, C V_{\text {inter-spot }}=29.29$ and $C V_{\text {inter-day }}=31.25$. The same pattern was shown for the TICp $\left(\mathrm{CV}_{\text {intra-spot }}=8.46, \mathrm{CV}_{\text {inter-spot }}=20.88\right.$ and $\left.\mathrm{CV}_{\text {inter-day }}=20.66\right)$ and pTIC $\left(\mathrm{CV}_{\text {intra-spot }}=7.99, \mathrm{CV}_{\text {inter-spot }}=19.91\right.$ and $\left.\mathrm{CV}_{\text {inter-day }}=19.30\right)$ 
methods (Figure 3). Additionally, data normalization allowed the reduction of $C V$ values at the three levels $(p<0.0001)$. Differences between both normalization methods (TICp and pTIC) varied between 0.47 for $\mathrm{CV}_{\text {intra-spot }}$ and 1.36 for $\mathrm{CV}_{\text {inter-day }}$, demonstrating that both allowed for reduced CV values at intra-spot $(44.80 \%$ and $47.94 \%$ for TICp and pTIC, respectively), inter-spot $(28.71 \%$ and $32.0 \%)$ and inter-day $(33.89 \%$ and $38.24 \%)$ levels. Reduced CV values were consistently recorded for VSE and VRE isolates alike (Table S3).

Table 1. List of common peaks $(n=18)$ found in the average spectra of the vancomycinresistant E. faecium and vancomycin-susceptible E. faecium isolates included in the classification set. $C V$ = coefficient of variation of intensity. Mean in intensity units.

\begin{tabular}{cccc}
\hline \multirow{2}{*}{ Mass $(\boldsymbol{m} / \boldsymbol{z})$} & & Measurements & \\
\cline { 2 - 4 } & Appearance (\%) & $\mathbf{C V ~ ( \% )}$ & Mean \\
\hline 2059.79 & $178 / 178(100)$ & 53.42 & 4361.20 \\
2188.53 & $178 / 178(100)$ & 56.61 & 5269.55 \\
2408.35 & $178 / 178(100)$ & 49.04 & 3316.45 \\
2537.05 & $178 / 178(100)$ & 53.06 & 3527.45 \\
2676.89 & $178 / 178(100)$ & 51.89 & 2170.47 \\
3026.71 & $178 / 178(100)$ & 47.65 & 1270.16 \\
3517.85 & $178 / 178(100)$ & 56.14 & 1327.19 \\
3673.77 & $178 / 178(100)$ & 50.43 & 3344.71 \\
4430.01 & $178 / 178(100)$ & 46.26 & $17,437.72$ \\
4532.78 & $178 / 178(100)$ & 57.94 & 2296.61 \\
5356.72 & $178 / 178(100)$ & 56.15 & 8719.83 \\
5974.65 & $178 / 178(100)$ & 68.28 & 2531.38 \\
6055.07 & $178 / 178(100)$ & 50.30 & 4187.74 \\
6830.35 & $178 / 178(100)$ & 71.56 & 3020.35 \\
6891.33 & $178 / 178(100)$ & 59.00 & 5252.80 \\
7035.47 & $178 / 178(100)$ & 65.96 & 2656.59 \\
7347.39 & $178 / 178(100)$ & 55.31 & 2826.29 \\
9062.75 & $178 / 178(100)$ & 66.94 & \\
\hline
\end{tabular}

\section{Average Coefficient of Variation of peak intensities}

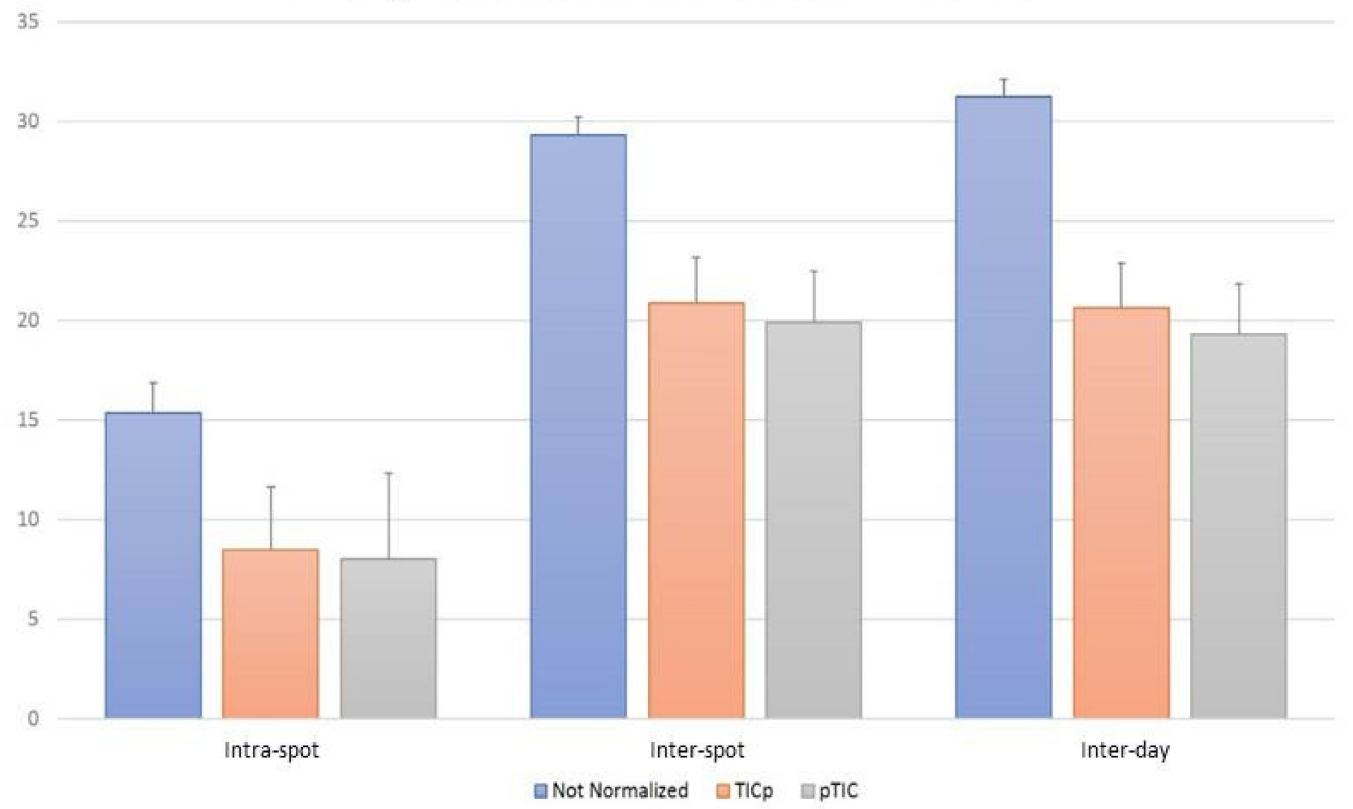

Figure 3. Coefficient of variation (CV) means for the intensity of the 18 common protein peaks of E. faecium analyzed at the intra-spot, inter-spot and inter-day levels using raw data (non-normalized) and both normalizations methods-before (TICp) and after (pTIC) finding peaks. 
The variability of the 18 common peaks found in all E. faecium isolates showed that CV values were lower for peaks between 3000 and $9000 \mathrm{~m} / \mathrm{z}$ at intra-spot (Figure 4A), interspot (Figure 4B) and inter-day levels (Figure 4C), especially when data were normalized, showing different means $(p<0.05)$ versus the 2000 and $3000 \mathrm{~m} / z$ range (Table S4). The $5974.6 \mathrm{~m} / z$ peak showed higher $\mathrm{CV}$ values at the three levels but the region between 6000 and $9000 \mathrm{~m} / z$ showed lower variation again, although the CVs were higher for interspot and inter-day repeatability (Figure 4B,C). These results support the fact that the central $\mathrm{m} / \mathrm{z}$ region of the spectrum is the most reliable for peak analysis [24].

A Intra-spot CV

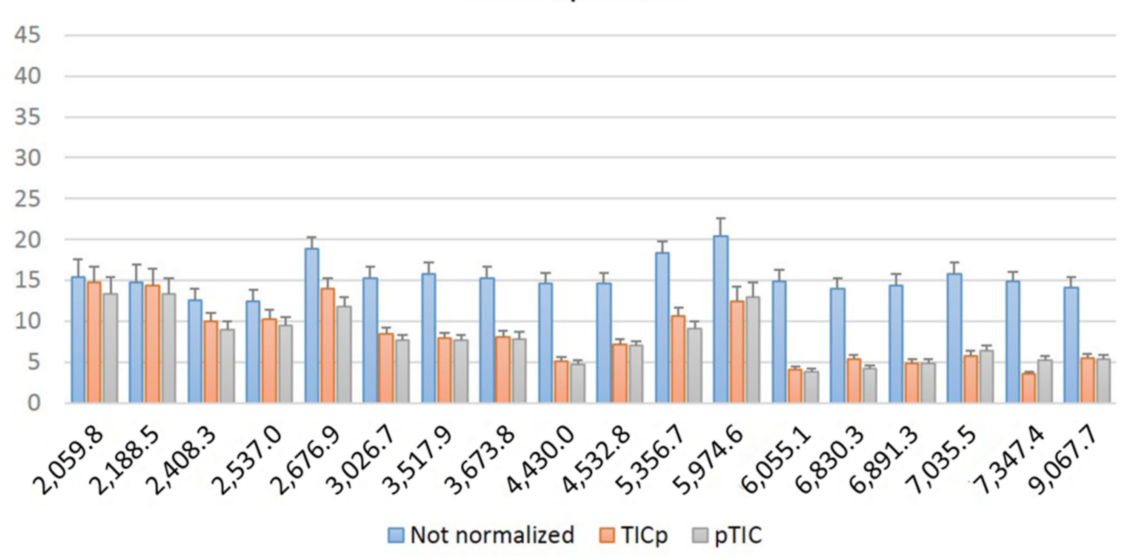

B Inter-spot CV
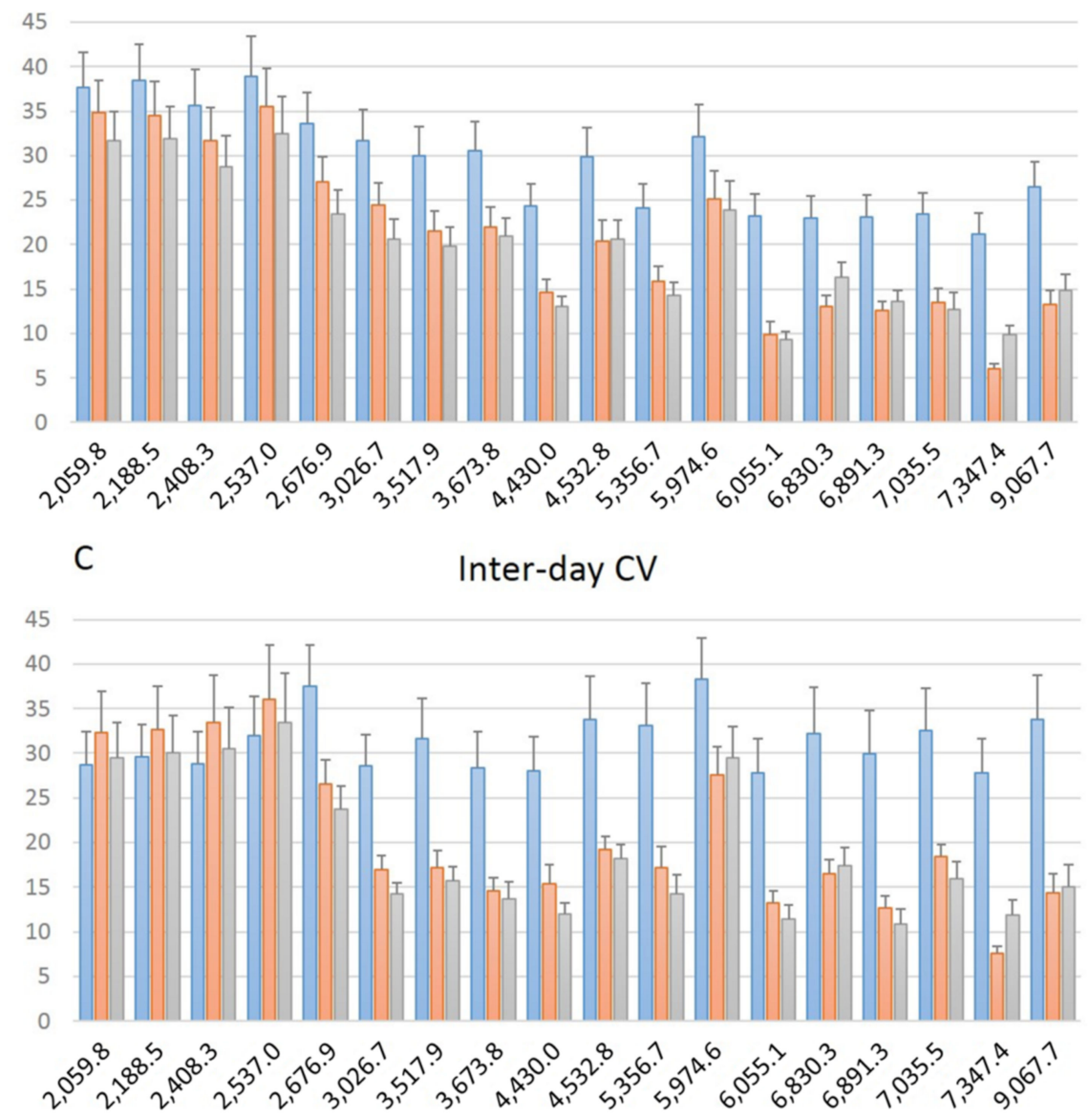

Figure 4. Comparison of the average coefficient of variation $(\mathrm{CV})$ for the peak intensity of the 18 common peaks analyzed at intra-spot (A), inter-spot (B) and inter-day (C) levels. 
The Pearson correlation coefficient was applied to inter-day repeatability, showing a mean of 0.94 factor for all samples (Table S2). This value was higher for VRE $(p=0.95)$ than for VSE isolates (0.93). Within the VRE group, the $p$ value for isolates hosting the vanA mechanism was 0.98 versus 0.92 for the isolates with the $\operatorname{vanB}$ resistance gene.

Arithmetic mean, as a representative value of peak intensity, and post-alignment mean were calculated and compared (Table 2). For non-normalized data, the arithmetic mean was higher than the post-alignment mean for inter-spot and inter-day levels, unlike the case for the TICp normalization method. Regarding pTIC normalization, the arithmetic and post-alignment means were identical. Furthermore, the homoscedasticity of the data and their normal distribution were checked by the implementation of the Levene and ShapiroWilk tests respectively. The t-Student test was then performed to check whether the null hypothesis of equality of means could be accepted among arithmetic and post-alignment means. In all possible comparisons, $p$-values obtained were $>0.05$ (Table S5), showing that, even when a post-alignment was performed, the intensity values were not affected. The fact that both means in all methods did not show statistical differences, proved the high repeatability of the assays based on protein spectra analysis.

Table 2. Arithmetic and post-alignment means at spot- and day-level without normalization and with the two different normalization methods applied—before (TICp) and after (pTIC) finding peaks.

\begin{tabular}{ccccccc}
\hline & \multicolumn{2}{c}{ Not Normalized } & \multicolumn{2}{c}{ TICp } & \multicolumn{2}{c}{ pTIC } \\
\cline { 2 - 7 } & Arithmetic & Post-Alignment & Arithmetic & Post-Alignment & Arithmetic & Post-Alignment \\
\hline Inter-spot mean & 3825.44 & 3792.14 & 0.0005 & 0.0005 & 0.056 & 0.056 \\
Inter-day mean & 3974.07 & 3955.49 & 0.0005 & 0.0005 & 0.056 & 0.056 \\
\hline
\end{tabular}

\subsection{Classification of the Isolates Using Machine Learning}

Three peak matrices generated (full-spectrum method and threshold methods TICp and pTIC) with data from the "classification set" were used as input data to test the capacity of the algorithms to discriminate VRE (VanA + VanB) from VSE isolates (Table S6). The 10 -fold cross validation results for the SVM algorithm showed the best accuracy with $80.9 \%$ and an F1 score (the harmonic mean of the sensitivity and the accuracy of the model) of $80.5 \%$ for the full-spectrum-TIC method (Table 3A).

Table 3. Discrimination of vancomycin-susceptible E. faecium (VSE) from vancomycin-resistant $E$. faecium (VRE) isolates and, within the latter group, differentiation between the strains hosting the van $A$ and $v a n B$ resistance genes. The actual classification is shown in columns and the predictive classification in rows. (A) Results from the support vector machine (SVM) algorithm using the full-spectrum-TIC method. Accuracy: 80.9\%; F1 score: $80.46 \%$; sensitivity: $81.4 \%$ specificity: $80.43 \%$; positive predictive value (PPV) or precision: $79.55 \%$; negative predictive value (NPV): $82.22 \%$. (B) Scores from the partial least squares-discrimination analysis (PLS-DA) algorithm using the total ion current normalization after peak finding (TICp) method. Accuracy: 86.05\%; predictive value for VanA VRE: $80.65 \%$; predictive value for VanB VRE: $89.09 \%$.

\begin{tabular}{ccc}
\hline Actual Classification & \multicolumn{2}{c}{ Predicted Classification } \\
\hline (A) SVM Full Spectrum & VRE & VSE \\
VRE & 70 & 16 \\
VSE & 18 & 74 \\
(B) PLS Threshold TICp & VanA & VanB \\
VanA & 25 & 6 \\
VanB & 6 & 49 \\
\hline
\end{tabular}

The same procedures were also applied for the discrimination of VanA from VanB VRE strains (Table S7). In this approach, PLS-DA algorithm with TICp method provided 
$86.65 \%$ correct classification (Figure 5). This algorithm achieved $89.09 \%$ of predictive value for identifying VanB VRE strains in a 10-fold cross validation (Table 3B).

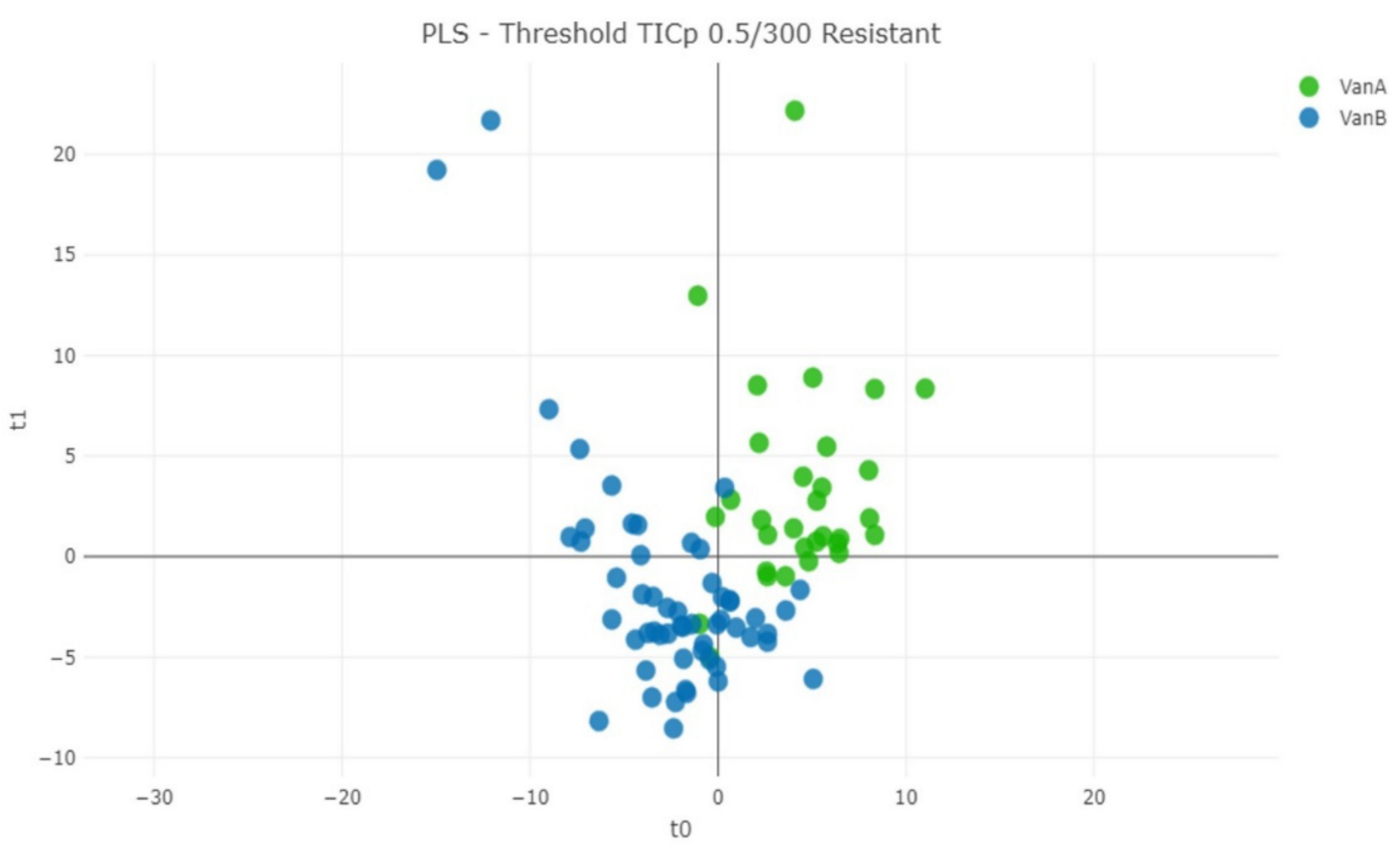

Figure 5. Distance plot of partial least squares-discrimination analysis (PLS-DA) machine learning algorithm for the discrimination of VanA from VanB VRE isolates using the TICp method.

The biomarker analysis revealed the presence of two potential resistance biomarkers at 6891.33 and $5095.01 \mathrm{~m} / \mathrm{z}$ (Table 4). These two peaks showed AUC values greater than 0.8 in its receiver operating characteristic (ROC) curve (Figure S1). The $5095.01 \mathrm{~m} / \mathrm{z}$ peak allowed the discrimination of the VRE strains from the VSE strains (Figure S2). This peak was present in 82 of the 86 VRE strains in this study. Furthermore, the AUC for peak at $6891.33 \mathrm{~m} / \mathrm{z}$ allowed the discrimination of VRE isolates hosting the vanA resistance mechanism from those carrying the vanB one. Its intensity was higher in all VanA E. faecium isolates tested with an AUC of 0.831 and a CV value of $22.99 \%$ (Figure S2). These results were obtained by applying a threshold ( 0.01 factor) after a TIC normalization using the 178 pre-processed samples of the classification set.

Table 4. Biomarker peaks for the detection of the vancomycin-resistant E. faecium (VRE) isolates and the discrimination of strains hosting the VanA mechanism.

\begin{tabular}{ccccc}
\hline Peak $m / z$ & AUC $(\geq \mathbf{0 . 8 )}$ & $\begin{array}{c}\text { Appearance } \\
\text { (Total Samples) }\end{array}$ & $\begin{array}{c}\text { Positive } \\
\text { Category }\end{array}$ & $\begin{array}{c}\text { Coefficient of } \\
\text { Variation (CV) }\end{array}$ \\
\hline 5095.01 & 0.814 & $123 / 178$ & $82 / 86$ (resistant) & $61.63 \%$ \\
\hline
\end{tabular}

\section{Discussion}

The application of MALDI-TOF MS coupled with data analysis was shown to be a reproducible methodology- $\mathrm{CV}$ values $\leq 20.88$ for normalized data-that allowed the discrimination of VRE (VanA + VanB) from VSE in $80.9 \%$ of the cases using SVM and the correct differentiation of $86.6 \%$ of the E. faecium VanB isolates from the VanA VRE isolates by PLS-DA. Specific peaks for the discrimination of the studied isolates were found: the $5095.01 \mathrm{~m} / \mathrm{z}$ peak was present in 82/86 VREs. Although this peak has already been described as a biomarker for VRE isolates [25], its real meaning is currently under debate. Brackmann et al. (2020) recently reported the sequencing of the $5095.01 \mathrm{~m} / \mathrm{z}$ protein and identified the protein hiracin, a secretory protein encoded by the hirJM79 gene, whose 
role in the vancomycin resistance mechanism remains unknown [26]. Although our study supports the value of the $5095.01 \mathrm{~m} / \mathrm{z}$ peak as a marker for VRE isolates, caution should be exercised when using this peak for VRE presence until further studies unravel its correlation with vancomycin resistance.

The $6891.33 \mathrm{~m} / \mathrm{z}$ peak was found as a biomarker for VanA VRE isolates. Both the AUC (0.831) and the CV values (22.99\%) indicate the uniqueness and repeatability of this marker. Although this peak had been related before with different clonal complexes and sequence types [17], its correlation with VanA VRE isolates had not been reported so far [27].

The repeatability study carried out with 20 E. faecium isolates (10 VSE, 5 VanA VRE and 5 VanB VRE) demonstrated that the lowest CV values for peak intensities were obtained for normalized data $(p<0.0001)$, regardless of the order in which normalization and peak finding is performed. Intra-spot repeatability showed the highest rate of repeatability (CV values ranging from 7.99 to 8.46 ), although the $\mathrm{CV}$ values for inter-spot and interday repeatability ranged between 19.91 and 20.88 for inter-spot variability and between 19.30 to 20.66 for inter-day repeatability. Similar CV values (6.5-17\%) have been reported in a study that evaluated the technical repeatability of MALDI-TOF MS for quantitative protein profiling [28]. Therefore, this methodology is considered reproducible and feasible for peak analysis, especially in the range of 3000-9000 m/z. In addition, 13 of the 18 most representative common peaks for all E. faecium and also both biomarker peaks for VRE vs. VSE and VanA VRE vs. VanB VRE differentiation are located within this spectrum range. The implementation of the methodology described in this study could provide standardization for data comparison with other studies analyzing antimicrobial resistance with MALDI-TOF MS.

Previous studies have shown the ability of MALDI-TOF MS to differentiate among E. faecium vancomycin-resistant high-risk clones, clonal complexes and sequence types with different success rates [16-18]. Differentiation between VRE and VSE isolates has also been reported. Griffin et al. reported $88.45 \%$ correct discrimination using SVM and $88.24 \%$ with the implementation of the genetic algorithm [25]. This algorithm also allowed a discrimination rate of $92.4 \%$ for VanA VRE from VSE isolates in the study developed by Nakano et al. [27]. In our case, the application of SVM, RF and PLS-DA algorithms provided $80.9 \%, 79.2 \%$ and $77.5 \%$ correct classification of VRE and VSE isolates using the full-spectrum method. Moreover, discrimination between VanA and VanB VRE isolates was achieved in $86.6 \%$ of the cases by applying the TICp method. Discrepancies in the peaks used for discrimination of the different E. faecium groups were detected with the two previous studies: none of the peaks included in the study by Nakano et al. were found relevant in our models; besides, only the $5095.01 \mathrm{~m} / \mathrm{z}$ peak $(5094.7 \mathrm{~m} / \mathrm{z}$ in Griffin et al.) was common to both studies for the discrimination of VRE from VSE. In our predictive models, the $6603 \mathrm{~m} / \mathrm{z}$ peak proposed by Griffin et al. for the discrimination of VanA from VanB VRE was not considered discriminative. Instead, the $6891.78 \mathrm{~m} / \mathrm{z}$ peak served this purpose in our study.

Although further studies are requested in order to clarify the role of the $5095.01 \mathrm{~m} / \mathrm{z}$ peak for the routine detection of VanB VRE isolates, we propose the detection of this peak in combination with the $6891.78 \mathrm{~m} / \mathrm{z}$ peak for the detection of suspected VanA VRE, for the differentiation of VRE using MALDI-TOF MS. Despite VanB VRE isolates being more prevalent in our setting, VanA VRE isolates have been reportedly correlated with hospital infections in different European countries [8]. Thus, both biomarker peaks could be used for rapid screening of VRE isolates with MALDI-TOF MS.

One of the limitations of this study is the lack of genomic background for the analyzed isolates. Only the genes encoding vancomycin resistance were targeted, and its presence or absence was confirmed by molecular methods. Although this information was useful for the development of predictive models, more comprehensive information about our VRE isolates could help obtain higher discrimination power from the applied algorithms and also explain the misclassifications from the current models. 
Another limitation of the study is that clonality of the isolates was only analyzed within a clinical and epidemiological approach. HGUGM is a tertiary hospital where clinical departments are in different wards, far from each other, and contact among them is sporadic —as each department has its own medical staff-so an outbreak affecting different departments is unlikely. The theory of non-clonality is also supported by Griffin et al., since the $5095.01 \mathrm{~m} / \mathrm{z}$ peak was also found in their study, performed in a different continent [25]. We acknowledge that clonality cannot be fully discarded as we lack a deep whole-genome sequencing approach. More accurate molecular methods are needed to rule out that the E. faecium isolates analyzed in this study belong to the same clone. However, our results support the importance of a previously described peak for differentiating VRE from VSE isolates and add a new specific biomarker for the discrimination of VanA VRE strains.

The classification accuracy of the applied algorithms has shown to be $<90 \%$ in all cases. Therefore, further studies with well-characterized isolates sourced from different geographic origins are needed to confirm the results obtained in this work and improve them if possible.

\section{Conclusions}

In conclusion, MALDI-TOF MS has demonstrated acceptable discrimination of E. faecium isolates beyond species assignment. Although further refining is requested and isolates from different clones and origins have to be included in predictive models in order to understand how they are discriminated by MALDI-TOF MS, protein profiling could become a suitable tool for the rapid detection of VRE in clinical microbiology laboratories. Its implementation could be key for the control of VRE isolates in hospital settings.

Supplementary Materials: The following supporting information can be downloaded at https:/ / www.mdpi.com/article/10.3390/diagnostics12020328/s1, Table S1: Description of the 178 E. faecium isolates included in this study; Table S2: E. faecium isolates included in the repeatability assay. Their Pearson correlation coefficient for inter-day (biological) repeatability is shown; Table S3: Coefficient of variation of the intensity means per level, normalization after (pTIC) and before (TICp) and antibiotic susceptibility; Table S4: Comparison of the $p$-values between average coefficient of variation of intensity means for peaks from 2000 to $3000 \mathrm{~m} / \mathrm{z}$ and 3000 to $9000 \mathrm{~m} / \mathrm{z}$ range; Table S5: $p$-Values for arithmetic and post-alignment means at the spot and day level with and without normalization; Table S6: Accuracy and F1-factor of the internal validation for the classification of vancomycin-resistant E. faecium (VRE) and vancomycin-susceptible E. faecium (VSE) strains as a metric for algorithms scores. Data obtained with the k-fold cross validation method, where $k=10$. Scaled data; Table S7: Accuracy of internal validation for the classification of VanA and VanB vancomycinresistant E. faecium (VRE) strains as metric for algorithms scores. Data obtained with the k-fold cross validation method, where $k=10$. Scaled data; Figure S1: Area under the curve (AUC) values for the two potential biomarkers found for (A) vancomycin-resistant E. faecium (VRE) isolates $(5095.01 \mathrm{~m} / \mathrm{z}$ ) and (B) for VanA VRE isolates $(6891.33 \mathrm{~m} / \mathrm{z}$ ); Figure S2: Visual discrimination of protein spectra from vancomycin-resistant E. faecium (VRE) (in red) and vancomycin-susceptible E. faecium (VSE) (green) according to the presence/absence of the peak at $5095.01 \mathrm{~m} / z$ (A). Differentiation of Van A (light blue) and VanB VRE isolates (purple) was based on the intensity of the peak at $6891.33 \mathrm{~m} / \mathrm{z}$ (B).

Author Contributions: A.C.: experimental part, formal analysis, data collection, validation, visualization, writing - original draft preparation and review/editing; M.J.A., Á.S.-M. and G.M., data analysis, validation, writing —original draft preparation and review/editing; D.R.-T., E.C. and M.M., formal analysis, data analysis and review/editing; L.Q. and A.R., experimental part and data collection; P.M., manuscript review; L.M., conceptualization, formal analysis, validation, original draft preparation and review/editing; B.R.-S., conceptualization, project administration, formal analysis, supervision, validation, visualization, original draft preparation and review/editing. All authors have read and agreed to the published version of the manuscript.

Funding: This work was supported by project PI15/01073 from the Health Research Fund (Instituto de Salud Carlos III. Plan Nacional de I + D + I 2013-2016) of the Carlos III Health Institute (ISCIII, Madrid, Spain) partially financed by the European Regional Development Fund (FEDER) “A way 
of making Europe". B.R.-S. (CPII19/00002) is a recipient of a Miguel Servet contract supported by the ISCIII.

Institutional Review Board Statement: The research presented in this study was conducted in accordance with the Declaration of Helsinki. It did not involve humans or human data. The Ethics Committee for Research with Medicinal Products from the Hospital Gregorio Marañón-CEIm (code no. MICRO.HGUGM.2020-002, 15 September 2020) approved this study. This committee determined that, since only bacterial isolates (not human products) were analyzed, all the conditions to waive the informed consent have been met.

Informed Consent Statement: Not applicable.

Data Availability Statement: All data generated or analyzed during this study are included in this published article (and its Supplementary Materials Files). Classification algorithms are available at https:/ / platform.clovermsdataanalysis.com/ accessed on 20 December 2021.

Acknowledgments: The authors are grateful to Luis Alcalá for his assistance with statistical analysis.

Conflicts of Interest: The authors declare no conflict of interest. The funders had no role in the design of the study; in the collection, analyses, or interpretation of data; in the writing of the manuscript; or in the decision to publish the results.

\section{References}

1. Vu, J.; Carvalho, J. Enterococcus: Review of its physiology, pathogenesis, diseases and the challenges it poses for clinical microbiology. Front. Biol. 2011, 6, 357-366. [CrossRef]

2. Arias, C.A.; Murray, B.E. The rise of the Enterococcus: Beyond vancomycin resistance. Nat. Rev. Microbiol. 2012, 10, 266-278. [CrossRef] [PubMed]

3. Cattoir, V.; Leclercq, R. Twenty-five years of shared life with vancomycin-resistant enterococci: Is it time to divorce? J. Antimicrob. Chemother. 2013, 68, 731-742. [CrossRef] [PubMed]

4. Miller, W.R.; Murray, B.E.; Rice, L.B.; Arias, C.A. Resistance in Vancomycin-Resistant Enterococci. Infect. Dis. Clin. N. Am. 2020, 34, 751-771. [CrossRef]

5. Cattoir, V.; Giard, J.C. Antibiotic resistance in Enterococcus faecium clinical isolates. Expert Rev. Anti-Infect. Ther. 2014, 12, 239-248. [CrossRef]

6. Rice, L.B. Federal funding for the study of antimicrobial resistance in nosocomial pathogens: No ESKAPE. J. Infect. Dis. 2008, 197, 1079-1081. [CrossRef]

7. Chiang, H.Y.; Perencevich, E.N.; Nair, R.; Nelson, R.E.; Samore, M.; Khader, K.; Chorazy, M.L.; Herwaldt, L.A.; Blevins, A.; Ward, M.A.; et al. Incidence and Outcomes Associated with Infections Caused by Vancomycin-Resistant Enterococci in the United States: Systematic Literature Review and Meta-Analysis. Infect. Control Hosp. Epidemiol. 2017, 38, 203-215. [CrossRef]

8. Lopez, M.; Cercenado, E.; Tenorio, C.; Ruiz-Larrea, F.; Torres, C. Diversity of clones and genotypes among vancomycin-resistant clinical Enterococcus isolates recovered in a Spanish hospital. Microb. Drug Resist. 2012, 18, 484-491. [CrossRef]

9. Mirzaei, B.; Farivar, T.N.; Juhari, P.; Mehr, M.A.; Babaei, R. Investigation of the Prevalence of vanA and vanB genes in vancomycin resistant enterococcus (VRE) by Taq Man real time PCR Assay. J. Microbiol. Infect. Dis. 2013, 3, 192. [CrossRef]

10. Hricová, K.; Štosová, T.; Kučová, P.; Fišerová, K.; Bardoň, J.; Kolář, M. Analysis of Vancomycin-Resistant Enterococci in Hemato-Oncological Patients. Antibiotics 2020, 9, 785. [CrossRef]

11. Seng, P.; Drancourt, M.; Gouriet, F.; La Scola, B.; Fournier, P.E.; Rolain, J.M.; Raoult, D. Ongoing revolution in bacteriology: Routine identification of bacteria by matrix-assisted laser desorption ionization time-of-flight mass spectrometry. Clin. Infect. Dis. Off. Publ. Infect. Dis. Soc. Am. 2009, 49, 543-551. [CrossRef]

12. Oviaño, M.; Gómara, M.; Barba, M.J.; Revillo, M.J.; Barbeyto, L.P.; Bou, G. Towards the early detection of beta-lactamase-producing Enterobacteriaceae by MALDI-TOF MS analysis. J. Antimicrob. Chemother. 2017, 72, 2259-2262. [CrossRef] [PubMed]

13. Schuster, D.; Josten, M.; Janssen, K.; Bodenstein, I.; Albert, C.; Schallenberg, A.; Gajdiss, M.; Sib, E.; Szekat, C.; Kehl, K.; et al. Detection of methicillin-resistant coagulase-negative staphylococci harboring the class A mec complex by MALDI-TOF mass spectrometry. Int. J. Med. Microbiol. 2018, 308, 522-526. [CrossRef] [PubMed]

14. Oviano, M.; Bou, G. Matrix-Assisted Laser Desorption Ionization-Time of Flight Mass Spectrometry for the Rapid Detection of Antimicrobial Resistance Mechanisms and Beyond. Clin. Microbiol. Rev. 2019, 32, 927-937.

15. Rodriguez-Sanchez, B.; Cercenado, E.; Coste, A.T.; Greub, G. Review of the impact of MALDI-TOF MS in public health and hospital hygiene, 2018. Eurosurveillance 2019, 24, 1800193. [CrossRef] [PubMed]

16. Lasch, P.; Fleige, C.; Stämmler, M.; Layer, F.; Nübel, U.; Witte, W.; Werner, G. Insufficient discriminatory power of MALDI-TOF mass spectrometry for typing of Enterococcus faecium and Staphylococcus aureus isolates. J. Microbiol. Methods 2014, 100, 58-69. [CrossRef] 
17. Freitas, A.R.; Sousa, C.; Novais, C.; Silva, L.; Ramos, H.; Coque, T.M.; Lopes, J.; Peixe, L. Rapid detection of high-risk Enterococcus faecium clones by matrix-assisted laser desorption ionization time-of-flight mass spectrometry. Diagn. Microbiol. Infect. Dis. 2017, 87, 299-307. [CrossRef]

18. Holzknecht, B.J.; Dargis, R.; Pedersen, M.; Pinholt, M.; Christensen, J.J.; Hammerum, A.M.; Littauer, P.; Worning, P.; Westh, H.; Moser, C.; et al. Typing of vancomycin-resistant enterococci with MALDI-TOF mass spectrometry in a nosocomial outbreak setting. Clin. Microbiol. Infect. Off. Publ. Eur. Soc. Clin. Microbiol. Infect. Dis. 2018, 24, 1104.e1-1104.e4. [CrossRef] [PubMed]

19. Rodriguez-Sanchez, B.; Marín, M.; Sánchez-Carrillo, C.; Cercenado, E.; Ruiz, A.; Rodríguez-Créixems, M.; Bouza, E. Improvement of matrix-assisted laser desorption/ionization time-of-flight mass spectrometry identification of difficult-to-identify bacteria and its impact in the workflow of a clinical microbiology laboratory. Diagn. Microbiol. Infect. Dis. 2014, 79, 1-6. [CrossRef] [PubMed]

20. Oberle, M.; Wohlwend, N.; Jonas, D.; Maurer, F.P.; Jost, G.; Tschudin-Sutter, S.; Vranckx, K.; Egli, A. The Technical and Biological Reproducibility of Matrix-Assisted Laser Desorption Ionization-Time of Flight Mass Spectrometry (MALDI-TOF MS) Based Typing: Employment of Bioinformatics in a Multicenter Study. PLoS ONE 2016, 11, e0164260. [CrossRef]

21. Quintela-Baluja, M.; Böhme, K.; Fernández-No, I.C.; Morandi, S.; Alnakip, M.E.; Caamaño-Antelo, S.; Barros-Velázquez, J.; Calo-Mata, P. Characterization of different food-isolated Enterococcus strains by MALDI-TOF mass fingerprinting. Electrophoresis 2013, 34, 2240-2250. [CrossRef] [PubMed]

22. Zvezdanova, M.E.; Arroyo, M.J.; Méndez, G.; Guinea, J.; Mancera, L.; Muñoz, P.; Rodríguez-Sánchez, B.; Escribano, P. Implementation of MALDI-TOF Mass Spectrometry and Peak Analysis: Application to the Discrimination of Cryptococcus neoformans Species Complex and Their Interspecies Hybrids. J. Fungi 2020, 6, 330. [CrossRef] [PubMed]

23. Weis, C.V.; Jutzeler, C.R.; Borgwardt, K. Machine learning for microbial identification and antimicrobial susceptibility testing on MALDI-TOF mass spectra: A systematic review. Clin. Microbiol. Infect. Off. Publ. Eur. Soc. Clin. Microbiol. Infect. Dis. 2020, 26, 1310-1317. [CrossRef] [PubMed]

24. Timm, W.; Scherbart, A.; Bocker, S.; Kohlbacher, O.; Nattkemper, T.W. Peak intensity prediction in MALDI-TOF mass spectrometry: A machine learning study to support quantitative proteomics. BMC Bioinform. 2008, 9, 443. [CrossRef] [PubMed]

25. Griffin, P.M.; Price, G.R.; Schooneveldt, J.M.; Schlebusch, S.; Tilse, M.H.; Urbanski, T.; Hamilton, B.; Venter, D. Use of matrixassisted laser desorption ionization-time of flight mass spectrometry to identify vancomycin-resistant enterococci and investigate the epidemiology of an outbreak. J. Clin. Microbiol. 2012, 50, 2918-2931. [CrossRef]

26. Brackmann, M.; Leib, S.L.; Tonolla, M.; Schurch, N.; Wittwer, M. Antimicrobial resistance classification using MALDI-TOF-MS is not that easy: Lessons from vancomycin-resistant Enterococcus faecium. Clin. Microbiol. Infect. Off. Publ. Eur. Soc. Clin. Microbiol. Infect. Dis. 2020, 26, 391-393. [CrossRef]

27. Nakano, S.; Matsumura, Y.; Kato, K.; Yunoki, T.; Hotta, G.; Noguchi, T.; Yamamoto, M.; Nagao, M.; Ito, Y.; Takakura, S.; et al. Differentiation of vanA-positive Enterococcus faecium from vanA-negative E. faecium by matrix-assisted laser desorption/ionisation time-of-flight mass spectrometry. Int. J. Antimicrob. Agents 2014, 44, 256-259. [CrossRef]

28. Pang, R.T.; Johnson, P.J.; Chan, C.M.; Kong, E.K.; Chan, A.T.; Sung, J.J.; Poon, T.C. Technical evaluation of MALDI-TOF mass spectrometry for quantitative proteomic profiling matrix formulation and application. Clin. Proteom. J. 2004, 1, 12. [CrossRef] 